\title{
Heat shock protein 27 promotes cell proliferation through activator protein-1 in lung cancer
}

\author{
SAI ZHANG ${ }^{1}$, YANGMIN HU ${ }^{2}$, YUWEN HUANG ${ }^{2}$, HUIMIN XU $^{2}$, GONGXIONG WU $^{3}$ and HAIBIN DAI ${ }^{2}$ \\ Departments of ${ }^{1}$ Thoracic Surgery and ${ }^{2}$ Pharmacy, Second Affiliated Hospital, Zhejiang University School of Medicine, \\ Hangzhou, Zhejiang 310009, P.R. China; ${ }^{3}$ Research Division, Joslin Diabetes \\ Center, Harvard Medical School, Boston, MA 02215, USA
}

Received June 9, 2014; Accepted March 5, 2015

DOI: $10.3892 / \mathrm{ol} .2015 .3073$

\begin{abstract}
Heat shock protein 27 (HSP27) is an important regulator involved in the development of lung cancer. However, limited evidence exists concerning the underlying molecular mechanisms of its action. The results of the present study revealed that HSP27 was highly expressed in the lung cancer tissues of mice. In an in vitro model, the overexpression of HSP27 promoted cell proliferation, while HSP27 knockdown inhibited cell proliferation. HSP27 promoted cell proliferation in vitro by directly upregulating the expression of HSP27 target genes, which required the activation of the activator protein-1 (AP-1) signaling pathway. This was evaluated by the phosphorylation status of an important pathway component, c-Jun in lung cancer tissue and cells. These results suggested that HSP27 has a promotional role in lung cancer, and therefore indicated a novel mechanism involving lung cancer cell proliferation, which may underlie poor responses to therapy. Therefore, HSP27 may be a suitable therapeutic target for the treatment of lung cancer.
\end{abstract}

\section{Introduction}

Lung cancer is the leading cause of cancer-associated mortality worldwide, with non-small cell lung cancer (NSCLC) accounting for $\sim 85 \%$ of all mortalities associated with lung cancer. The overall five-year survival rate for patients remains poor (1-3). The poor prognoses of lung cancer patients primarily result from early relapse, metastasis and unsuccessful responses to treatment strategies, including surgery, chemotherapy and radiotherapy (4-6). In addition, the lack of effective prognostic biomarkers that are able to predict treatment response and prognosis affects treatment regimens and patient outcomes.

Heat shock proteins (HSPs) are a large family of proteins that function as molecular chaperones under physiological

Correspondence to: Professor Haibin Dai, Department of Pharmacy, Second Affiliated Hospital, Zhejiang University School of Medicine, 88 Jiefang Road, Hangzhou, Zhejiang 310009, P.R. China E-mail: haibindai@zju.edu.cn

Key words: lung cancer, proliferation, heat shock protein 27, activator protein-1, c-Jun conditions (7). The ability of radiofrequency ablation to induce the expression of HSPs, including HSP70, in small animal models suggests that HSPs may be involved in rescuing damaged cells in close proximity to the ablation zone and, therefore, limiting the therapeutic effects $(8,9)$. In addition, HSPs have been identified to promote carcinogenesis by inhibiting apoptosis (10-14) and enhancing resistance to treatment $(15,16)$. However, it has been established that HSPs function differently in different tumors $(10,15)$.

HSP27 is a $27-\mathrm{kDa}$ protein that regulates apoptosis by interacting with key components of apoptotic signaling pathways, particularly those involved in the activation of caspases. Increasing evidence has indicated that HSP27 may play an important role in cancer (17). The expression of HSP27 has been associated with poor prognoses in ovarian (18), breast $(10,19)$, gastric (20) and prostate cancers $(21)$, as well as in osteoscarcomas (14). In addition, HSP27 has been reported to be associated with poor prognoses in patients with lung cancer (22). However, whether the expression of HSP27 has a prognostic role in lung cancer remains controversial. Zimmermann et al (22) reported that the level of serum HSP27 was positively correlated with advanced lung cancer stages (22). Further studies demonstrated that HSP27 enhanced the chemoresistance of NSCLC, which is a poor indicator of prognosis $(16,23)$.

The present study aimed to investigate the expression of HSP27 in the lung cancer tissues of mice and its association with cell proliferation and the activator protein-1 (AP-1) signaling pathway in vitro and in vivo. In addition, the potential role of HSP27 as a therapeutic target for the treatment of lung cancer was examined.

\section{Materials and methods}

Lung cancer mouse model. All the animal studies were performed in accordance with the regulations and approval of the Institutional Animal Care and Use Committee of Zhejiang University (Hangzhou, China; protocol no. ZJU201410-1-02-090), which were obtained prior to the initiation of the study. Athymic immunodeficient nude mice (BALB/c; n=10; age, 4 weeks; Shanghai Laboratory Animal Center, Shanghai, China) were maintained in pathogen-free facilities. The mice were subcutaneously implanted with $8 \times 10^{6}$ NCI-H82 small cell lung cancer 
cells (American Type Culture Collection, Rockville, MD, USA), which were left to grow for 2 weeks to a volume of $80-100 \mathrm{~mm}^{3}$. After the mice were sacrificed, tumors were extirpated and frozen in liquid nitrogen prior to storage at $-80^{\circ} \mathrm{C}$ for protein analysis. Control samples were obtained from healthy BALB/c mice (Shanghai Laboratory Animal Center).

Reagents. Small interfering RNA (siRNA) was purchased from Dharmacon Inc. (Lafayette, CO, USA). The primary antibodies used in the present study included rabbit phospho-c-Jun (p-c-Jun; Ser63) monoclonal antibody (dilution, 1:1,000; catalog no. 9261; Cell Signaling Technology Inc., Danvers, MA, USA), rabbit c-Jun polyclonal antibody (dilution, 1:1,000; catalog no. 9165, Cell Signaling Technology Inc.), and HSP27 polyclonal antibody (dilution, 1:1,000; catalog no. SPA-803, Stressgen, Chicago, IL, USA). Expression of the proliferative marker Ki-67 was performed to assess the growth of the lung cancer cells. The monoclonal mouse anti-human Ki-67 antibody was obtained from Thermo Fisher Scientific, (Rockford, IL, USA; catalog no. MA1-80199; dilution, 1:100).

Cell culture. The human lung adenocarcinoma A549 cells (American Type Culture Collection) were cultured in Dulbecco's modified Eagle's medium (DMEM) supplemented with $10 \%$ fetal bovine serum (FBS; Gibco Life Technologies, Carlsbad, CA, USA). The cells were maintained at $37^{\circ} \mathrm{C}$ in a $5 \% \mathrm{CO}_{2}$ humidified atmosphere.

Bromodeoxyuridine incorporation assay. Cells were seeded on coverslips (Fisher, Pittsburgh, PA, US) in 24-well plates $\left(5 \times 10^{4}\right.$ cells/plate). After $24 \mathrm{~h}$, the cells were incubated with Bromodeoxyuridine (BrdU) for $1 \mathrm{~h}$, and stained with anti-BrdU antibody (Upstate, Temecula, CA, US), following the manufacturer's instructions. Gray level images were acquired under a laser scanning microscope (Axioskop 2 plus; Carl Zeiss Co. Ltd., Jena, Germany).

siRNA transfection. The human lung adenocarcinoma A549 cells were grown in antibiotic-free DMEM (Gibco Life Technologies) supplemented with 10\% FBS. Upon reaching $70 \%$ confluence, the cells were transfected with a negative control siRNA or HSP27 siRNA. Lipofectamine ${ }^{\circledR}$ transfection reagent (Invitrogen Life Technologies, Carlsbad, CA, USA) was used for the transfections, according to the manufacturer's instructions. The final siRNA concentration was $20 \mathrm{nmol} / 1$ for each transfection. After $48 \mathrm{~h}$, the cells were collected and subjected to further analysis.

Western blot analysis. Fresh tumor samples harvested from mice were homogenized and centrifuged at $1,000 \mathrm{x} \mathrm{g}$ for 5 min. The cell pellets were then washed with phosphate-buffered saline (PBS) and lysed using a reducing Laemmli sample buffer supplemented with protease inhibitors (Sigma-Aldrich, St. Louis, MO, USA). Next, the proteins were separated on 4-20\% Tris-glycine gels and transferred onto a polyvinylidene difluoride membrane. Non-specific binding sites were blocked by incubating the membrane for $1 \mathrm{~h}$ in PBS and Tween 20 with $5 \%$ nonfat milk. The blots were probed with a primary antibody corresponding to the protein of interest, or with a rabbit anti-mouse $\beta$-actin polyclonal antibody (1:2,000 dilution; catalog no. 4967, Cell Signaling Technology) that was used as the loading control. Subsequent to washing and incubating with secondary horseradish peroxidase (HRP)-conjugated antibodies (Abcam, Cambridge, MA, USA), the membranes were developed using an Immobilon Western Chemiluminescent HRP Substrate (EMD Millipore, Billerica, MA, USA). The protein content was measured using densitometry analysis (Image J; NIH Image, Bethesda, MD, USA) and normalized against the $\beta$-actin protein content.

Quantitative real time polymerase chain reaction ( $q P C R)$ analysis. According to a previous study (24), total RNA was isolated from the mouse tumor tissues or cultured cells using the RNeasy Plus Mini kit (Qiagen, Santa Clarita, CA, USA). qPCR was performed in an ABI Prism 7000 Sequence Detection System (Applied Biosystems, Foster City, CA, USA) using SYBR Green in an iCycler (Bio-Rad Laboratories Inc., Hercules, CA, USA). The following primers were used to detect the Hsp27 mRNA levels: Forward, 5'-TCCCTGGATGTC AACCACTTCG-3', and reverse, 5'-GGGACAGGGAGGAGG AAACTTG-3'. 18S ribosomal RNA was used as the internal control; Forward, 5'-CAACACGGGAAATCTCACCA-3', and reverse, 5'-GACAAATCGCTCCACCAACTAA-3'. PCR was performed under the following conditions: Amplification for $10 \mathrm{sec}$ at $95^{\circ} \mathrm{C}$, followed by 40 cycles of $95^{\circ} \mathrm{C}$ for $5 \mathrm{sec}$ and $60^{\circ} \mathrm{C}$ for $30 \mathrm{sec}$. Data were collected after each annealing step and the relative expression of genes was calculated using the $2^{-\Delta \Delta \mathrm{Ct}}$ method, as previously described (24).

Reporter gene assay. Cells were harvested in ice-cold PBS and collected by brief centrifugation at $12,000 \mathrm{x} \mathrm{g}$ for $10 \mathrm{~min}$. Cell lysates were obtained for the firefly luciferase assay according to the manufacturer's instructions (Promega, Madison, WI, USA). The luciferase activity was quantified using an Opticomp Luminometer (MGM Instruments, Hamden, CT, USA). For the $\beta$-galactosidase reporter gene assay, $30 \mu \mathrm{l}$ cell extract was mixed with $3 \mu \mathrm{l} 100 \mathrm{x} \mathrm{Mg}^{2+}$ solution ( $0.1 \mathrm{M} \mathrm{MgCl} 2$ and $4.5 \mathrm{M} \beta$-mecaptoethanol, $66 \mu 1$ $4 \mathrm{mg} / \mathrm{ml} O$-nitrophenyl- $\beta$-D-galactopyranoside in $0.1 \mathrm{M}$ sodium phosphate, $\mathrm{pH} 7.5$ and $201 \mu \mathrm{l} 0.1 \mathrm{M}$ sodium phosphate, $\mathrm{pH} 7.5$ ). The mixture was incubated at $37^{\circ} \mathrm{C}$ for $30 \mathrm{~min}$, the optical density at $405 \mathrm{~nm}$ was determined and expressed as the relative $\beta$-galactosidase activity. The $\beta$-galactosidase activity was subsequently used to normalize the transfection variations for the luciferase reporter gene assay.

Statistical analysis. Statistical analysis was performed using SPSS software (version 11; SPSS Inc., Chicago, IL, USA). The data are expressed as the mean \pm standard error of the mean. Statistical comparisons were performed using the Student's $t$-test between two groups. The significance of the differences compared with the untreated control for each group was assessed using analysis of variance (ANOVA). The ANOVA significance was confirmed by the $\mathrm{F}$ test. In all cases, $\mathrm{P}<0.05$ was used to indicate a statistically significant difference.

\section{Results}

HSP27 is highly expressed in lung cancer in vivo and in vitro. In order to investigate the role of HSP27 in lung cancer, the 
A

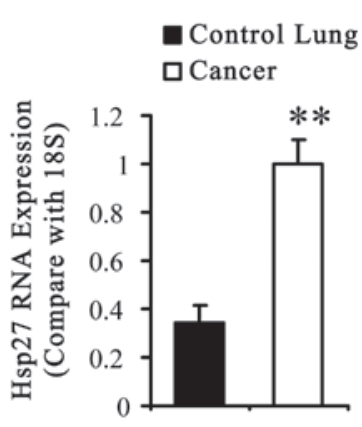

C

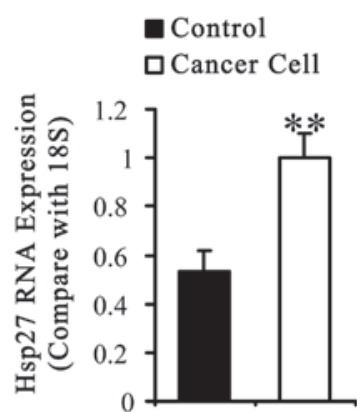

B

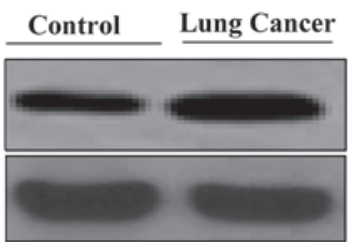

Hsp27

$\beta$-actin

D

Control Cancer cells

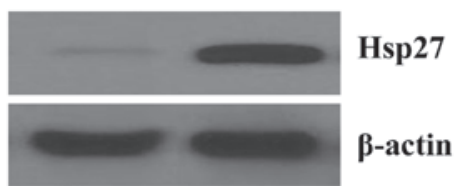

Figure 1. HSP27 was highly expressed in lung cancer tissues in vivo and lung cancer cells in vitro. In the lung cancer and normal tissues, (A) the mRNA expression of HSP27 was determined using RT-qPCR ( $\mathrm{n}=5$ ), while (B) the protein expression of HSP27 was determined using western blot analysis ( $\mathrm{n}=5$ ). In the lung cancer and non-lung cancer (control) cell lines, (C) the mRNA expression of HSP27 mRNA was measured using RT-qPCR (n=4), while (D) the protein expression of HSP27 was measured using western blot analysis $(n=4)$. $\beta$-actin was used as the loading control. ${ }^{* *} \mathrm{P}<0.01$, vs. control. HSP27, heat shock protein 27; RT-qPCR, reverse transcription-quantitative polymerase chain reaction.

A

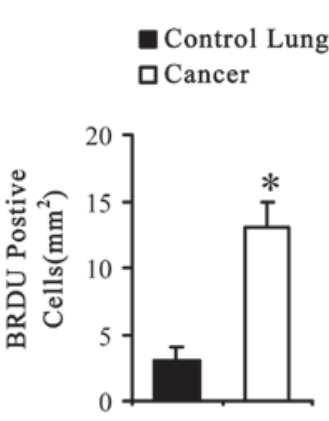

C

- Hsp27 siRNA

口Control siRNA

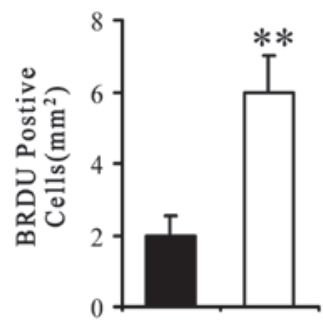

B

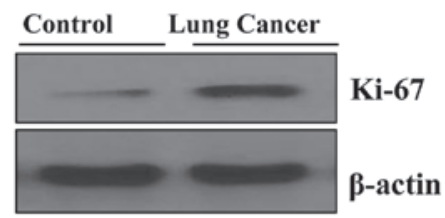

D

\section{Hsp27 siRNA Control siRNA}

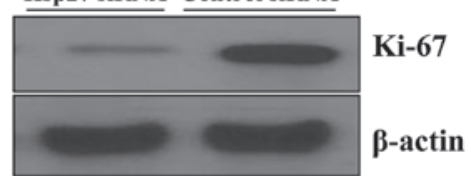

Figure 2. HSP27 promoted cell proliferation in lung cancer. (A) BrdU staining revealed positive cell proliferation in the cell line with a stable overexpression of HSP27 and in the control cell line. (B) Western blot analysis revealed the expression of Ki-67 in the cell line with a stable overexpression of HSP27 (HSP27 siRNA) and in the control cell line. (C) BrdU staining revealed positive cell proliferation in the HSP27-knowdown and control cells. (D) Western blot analysis revealed the expression of Ki-67 in HSP27-knowdown and control cells ( $\mathrm{n}=4$ per group). $\beta$-actin was used as the loading control. " $\mathrm{P}<0.05$ and ${ }^{* *} \mathrm{P}<0.01$, vs. control. BrdU, 5-bromo-2'-deoxyuridine; HSP27, heat shock protein 27; siRNA, small interfering RNA. 
A

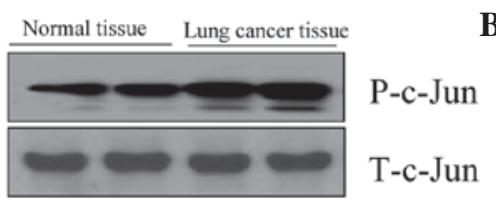

B

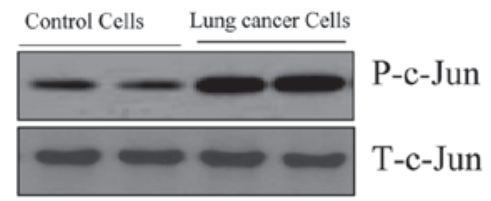

C

- Control Cells 口Hsp27 Cells

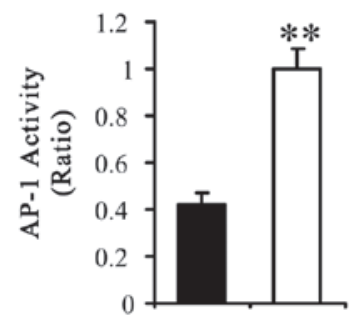

Figure 3. HSP27-induced cell proliferation depends on AP-1 signaling. (A) Western blot analysis demonstrating the phosphorylation of c-Jun in lung cancer and healthy control tissues. (B) Western blot analysis revealed the phosphorylation of c-Jun in the lung cancer and control cell lines. (C) Reporter gene assay revealed AP-1 activity in the lung cancer cell line with a stable overexpression of HSP27 and in the control cell line ( $\mathrm{n}=4$ per group). ${ }^{*} \mathrm{P}<0.05$ and ${ }^{* *} \mathrm{P}<0.01, \mathrm{vs}$. control. HSP27, heat shock protein 27; AP-1, activator protein-1; p-c-Jun, phospho-c-Jun; T-c-Jun, total c-Jun.

expression of HSP27 was analyzed in 1-month-old lung cancer mice and cultured A549 cells using RT-qPCR and western blot analyses. The expression of HSP27 was significantly higher in the peripheral areas of the lung cancer tissue compared with the normal tissue (Fig. 1A and B). These results suggest that an increased expression of HSP27 may contribute to lung cancer development. In order to confirm this finding, the expression of HSP27 in a lung cancer cell line and a non-lung cancer cell line was investigated. The expression of HSP27 was significantly higher in the lung cancer cell line compared with that in the non-lung cancer cell line (Fig. 1C and D). Therefore, the high expression of HSP27 may be a potential factor involved in the development of lung cancer.

HSP27 expression promotes cell proliferation in lung cancer. The present study also investigated the mechanism underlying the role of HSP27 in lung cancer development. A cell line with a stable overexpression of HSP27 was used to study the function of HSP27 in cell proliferation. The results of the 5-bromo-2'-deoxyuridine (BrdU) and Ki-67 blot assays revealed a significant increase in the proliferation of the HSP27-overexpressing cell line compared with the control cell line (Fig. 2A and B). By contrast, according to the BrdU and Ki-67 blot assays, knockdown of HSP27 by siRNA significantly inhibited cell proliferation (Fig. 2C and D). These data suggest that HSP27 expression promoted cell proliferation in lung cancer.

HSP27 expression promotes cell proliferation through the AP-1 signaling pathway. In order to assess the effect of HSP27 on the signaling pathways known to regulate HSP27 activity, the present study analyzed the expression levels of the AP-1 components, c-Jun and p-c-Jun, in the tumor samples. Western blot analysis revealed that there were significantly higher levels of p-c-Jun in the lung cancer tissues compared with the normal tissues. By contrast, the total levels of c-Jun were not altered in either group (Fig. 3A). This indicates that the activation of these signaling pathways may be important in regulating the outcome and efficacy of cancer combination therapies in tumor models. In accordance with these findings, a significant increase in the in vitro expression levels of p-c-Jun were observed in the human lung cancer cells compared with the control cells (Fig. 3B). These data indicate that HSP27-induced cell proliferation may occur as a result of the activation of AP-1 signaling.
In order to directly evaluate whether HSP27 regulates the activity of downstream AP-1 components, the present study used a firefly luciferase reporter gene assay to measure any effects that HSP27 had on the transcriptional output in lung cancer cells. The reporter gene activity under investigation was directly under the control of AP-1 response elements. The results indicate that the overexpression of HSP27 in tumor cells regulates transcriptional output through an upregulation in the activity of AP-1 (Fig 3C). Therefore, these findings present a novel HSP27 and AP-1 signaling pathway that regulates the therapeutic efficacy in the lung cancer treatment model. This novel pathway provides an opportunity to design improved therapeutic strategies for the treatment of cancer.

\section{Discussion}

HSP27 is an important regulator involved in the development of lung cancer. However, the precise mechanisms underlying its action are yet to be elucidated. The present study revealed that HSP27 was highly expressed in lung cancer tissues from mice, as well as in a lung cancer cell line. In the in vitro model, the overexpression of HSP27 promoted cell proliferation by upregulating specific target genes, which required the activation of the AP-1 signaling pathway following the phosphorylation of c-Jun. These results indicated that HSP27 may be a novel therapeutic target for the treatment of lung cancer.

HSPs are known to be key factors in the heat shock response and have been identified to be implicated in the etiology of breast cancer (7). The cellular levels of HSP27 are significantly increased in breast cancer cells compared with surrounding healthy tissues. These changes result in the HSP-mediated inhibition of pathways involved in apoptosis and replicative senescence, which is believed to lead to the growth of tumors and poor response to chemotherapy.

Increasing evidence has revealed that HSPs are overexpressed in a range of human carcinomas (25), including solid tumors and hematological malignancies $(17,26,27)$. A previous study reported a 3-fold increase in the expression levels of HSP27 in lung cancer tumor samples from patients (22). In addition, Bhardwai et al (8) and Solazzo et al (9) demonstrated in their mechanistic studies that HSPs, including HSP27, are induced in the development of lung cancer $(13,28)$. Consistent with the findings of previous studies, the present study demonstrated that the level of HSP27 was significantly higher in the mouse lung 
cancer model compared with normal cells, and was associated with enhanced cell proliferation and inhibited apoptosis. These results suggest that HSP27 may be a potential therapeutic target used to increase the positive clinical outcome of tumors.

To the best of our knowledge, the present study was the first to report that HSP27 promoted cell proliferation in lung cancer. This is consistent with the findings reported by Turakhia et al (29), which indicated that HSP27 reduced the doxorubicin-induced toxicity in cardiac cells by enhancing superoxide dismutase activity, and therefore protected the integrity of aconitases from superoxide-induced inactivation (29). According to the results of previous studies, the inhibition of HSPs has been established to have an impact on the growth of several malignant cell lines, including breast cancer (30) and leukemia (31). Furthermore, HSPs are known to form a complex with mutant $\mathrm{p} 53$, which allows tumor cells to bypass normal mechanisms of the cell cycle arrest. This has been associated with shorter disease-free survival (32) and chemotherapy drug resistance (33) in breast cancer. In the lung cancer tumor model of the current study, the HSP27-mediated lung cancer development is, in part, due to the subsequent activation of its downstream effector protein, AP-1. In conclusion, the AP-1 pathway is hypothesized to be a potential target for the development of novel cancer treatments that can be combined with existing therapies.

\section{Acknowledgements}

This study was supported by grants from the National Natural Science Foundation of China (nos. 81173040, 81373391 and 81302747), the Foundation from the Health Bureau of Zhejiang Province (nos. 2011KYA065 and 2012RCA027), the Zhejiang Province Traditional Chinese Medicine Foundation of China (nos. 2011ZZ009 and 2012ZB091) and the Foundation of Science and Technology Department of Zhejiang Province (no. 2014C33185).

\section{References}

1. Ferlay J, Shin HR, Bray F, et al: Estimates of worldwide burden of cancer in 2008: GLOBOCAN 2008. Int J Cancer 127: 2893-2917,2010.

2. Jemal A, Siegel R, Xu J and Ward E: Cancer statistics, 2010. CA Cancer J Clin 60: 277-300, 2010.

3. Zhao GY, Ding JY, Lu CL, Lin ZW and Guo J: The overexpression of 14-3-3६ and Hsp27 promotes non-small cell lung cancer progression. Cancer 120: 652-663, 2014.

4. Rózsás A,Berta J, Rojkó L, et al: Erythropoietin receptor expression is a potential prognostic factor in human lung adenocarcinoma. PLoS One 8: e77459, 2013.

5. Chen X, Liu Y, Røe OD, et al: Gefitinib or erlotinib as maintenance therapy in patients with advanced stage non-small cell lung cancer: a systematic review. PLoS One 8: e59314, 2013.

6. Tsai JR, Liu PL, Chen YH, et al: Ginkgo biloba extract decreases non-small cell lung cancer cell migration by downregulating metastasis-associated factor heat-shock protein 27. PLoS One 9: e91331, 2014

7. Wang X, Chen M, Zhou J and Zhang X: HSP27, 70 and 90, anti-apoptotic proteins, in clinical cancer therapy (Review). Int J Oncol 45: 18-30, 2014

8. Bhardwaj N, Dormer J, Ahmad F, et al: Heat shock protein 70 expression following hepatic radiofrequency ablation is affected by adjacent vasculature. J Surg Res 173: 249-257, 2012

9. Solazzo SA, Ahmed M, Schor-Bardach R, et al: Liposomal doxorubicin increases radiofrequency ablation-induced tumor destruction by increasing cellular oxidative and nitrative stress and accelerating apoptotic pathways. Radiology 255 62-74, 2010.
10. Ciocca DR and Calderwood SK: Heat shock proteins in cancer: diagnostic, prognostic, predictive and treatment implications. Cell Stress Chaperones 10: 86-103, 2005.

11. Erkizan O, Kirkali G, Yörükoğlu K and Kirkali Z: Significance of heat shock protein-27 expression in patients with renal cell carcinoma. Urology 64: 474-478, 2004.

12. Eskenazi AE, Powers J, Pinkas J, et al: Induction of heat shock protein 27 by hydroxyurea and its relationship to experimental metastasis. Clin Exp Metastasis 16: 283-290, 1998.

13. Moon A, Bacchini P, Bertoni F, et al: Expression of heat shock proteins in osteosarcomas. Pathology 42: 421-425, 2010.

14. Bausero MA, Page DT, Osinaga E and Asea A: Surface expression of Hsp25 and Hsp72 differentially regulates tumor growth and metastasis. Tumour Biol 25: 243-251, 2004.

15. Conroy SE, Sasieni PD, Amin V, et al: Antibodies to heat-shock protein 27 are associated with improved survival in patients with breast cancer. Br J Cancer 77: 1875-1879, 1998.

16. Hsu HS, Lin JH, Huang WC, et al: Chemoresistance of lung cancer stemlike cells depends on activation of Hsp27. Cancer 117: 1516-1528, 2011.

17. Concannon CG, Gorman AM and Samali A: On the role of Hsp27 in regulating apoptosis. Apoptosis 8: 61-70, 2003.

18. Arts HJ, Hollema H, Lemstra W, et al: Heat-shock-protein-27 (hsp27) expression in ovarian carcinoma: relation in response to chemotherapy and prognosis. Int J Cancer 84: 234-238, 1999.

19. Lemieux P, Oesterreich S, Lawrence JA, et al: The small heat shock protein hsp27 increases invasiveness but decreases motility of breast cancer cells. Invasion Metastasis 17: 113-123, 1997.

20. Kapranos N, Kominea A, Konstantinopoulos PA, et al: Expression of the 27-kDa heat shock protein (HSP27) in gastric carcinomas and adjacent normal, metaplastic and dysplastic gastric mucosa and its prognostic significance. J Cancer Res Clin Oncol 128: 426-432, 2002.

21. Cornford PA, Dodson AR, Parsons KF, et al: Heat shock protein expression independently predicts clinical outcome in prostate cancer. Cancer Res 60: 7099-7105, 2000.

22. Zimmermann M, Nickl S, Lambers C, et al: Discrimination of clinical stages in non-small cell lung cancer patients by serum HSP27 and HSP70: a multi-institutional case-control study. Clin Chim Acta 413: 1115-1120, 2012.

23. Berrieman HK, Cawkwell L, O'Kane SL, et al: Hsp27 may allow prediction of the response to single-agent vinorelbine chemotherapy in non-small cell lung cancer. Oncol Rep 15: 283-286, 2006.

24. Dai H, Yu Z, Fan X, et al: Dysfunction of annexin A2 contributes to hyperglycaemia-induced loss of human endothelial cell surface fibrinolytic activity. Thromb Haemost 109: 1070-1078, 2013

25. Soo ET, Yip GW, Lwin ZM, et al: Heat shock proteins as novel therapeutic targets in cancer. In Vivo 22: 311-315, 2008.

26. Whitesell L and Lindquist SL: HSP90 and the chaperoning of cancer. Nat Rev Cancer 5: 761-772, 2005.

27. Neckers L: Heat shock protein 90: the cancer chaperone. J Biosci 32: 517-530, 2007.

28. Marinova DM, Slavova YG, Trifonova N, et al: Stress protein Hsp27 expression predicts the outcome in operated small cell lung carcinoma and large cell neuroendocrine carcinoma patients. J BUON 18: 915-920, 2013.

29. Turakhia S, Venkatakrishnan CD, Dunsmore $\mathrm{K}$, et al: Doxorubicin-induced cardiotoxicity: direct correlation of cardiac fibroblast and $\mathrm{H} 9 \mathrm{c} 2$ cell survival and aconitase activity with heat shock protein 27. Am J Physiol Heart Circ Physiol 293: H3111-H3121, 2007.

30. Borie F, Bouvier AM, Herrero A, et al: Treatment and prognosis of hepatocellular carcinoma: a population based study in France. J Surg Oncol 98: 505-509, 2008.

31. Elia G, Amici C, Rossi A and Santoro MG: Modulation of prostaglandin A1-induced thermotolerance by quercetin in human leukemic cells: role of heat shock protein 70. Cancer Res 56: 210-217, 1996

32. Ciocca DR, Clark GM, Tandon AK, Fuqua SA, Welch WJ and McGuire WL: Heat shock protein hsp70 in patients with axillary lymph node-negative breast cancer: prognostic implications. J Natl Cancer Inst 85: 570-574, 1993.

33. Oesterreich S, Weng CN, Qiu M, Hilsenbeck SG, Osborne CK and Fuqua SA: The small heat shock protein hsp27 is correlated with growth and drug resistance in human breast cancer cell lines. Cancer Res 53: 4443-4448, 1993. 